\title{
Status and discovery prospects for light pseudoscalars in the NMSSM
}

\section{R. Aggleton}

University of Southampton, UK

Rutherford Appleton Laboratory, UK

Bristol university, $U K$

E-mail: robin.aggleton@cern.ch

\section{Barducci}

SISSA and INFN, Sezione di Trieste, via Bonomea 265, 34136 Trieste, Italy

E-mail: daniele.barducci@lapth.cnrs.fr

\section{N.-E. Bomark*}

University of Agder, Norway

E-mail: nilseb@uia.no

\section{S. Moretti}

University of Southampton, UK

E-mail: S.Morettidsoton.ac.uk

\section{Shepherd-Themistocleous}

University of Southampton, UK

Rutherford Appleton Laboratory, UK

E-mail: Claire.Shepherdestfc.ac.uk

\begin{abstract}
While most BSM searches at the LHC focus on heavy new states, the NMSSM contains the possibility of new light states that have escaped detection due to their singlet nature.

Here we focus on light pseudoscalars, investigating the parameter space impact of recent LHC searches for such light states stemming from the decay of the $125 \mathrm{GeV}$ Higgs boson. It is shown that, though direct searches can not yet compete with the requirement of the $125 \mathrm{GeV}$ scalar having SM-like couplings, the searches are touching the allowed parameter space and should make a phenomenological impact in the near future.
\end{abstract}

EPS-HEP 2017, European Physical Society conference on High Energy Physics 5-12 July 2017

Venice, Italy

${ }^{*}$ Speaker. 


\section{Introduction}

Five years after the discovery of the Higgs-boson, we have seen nothing but Standard Model (SM) physics from the LHC. So should we expect anything more, and if so, what?

The only problem we have that is really pointing towards new physics at the electroweak (EW) scale, is the hierarchy problem; other problems, like dark matter, may have its solution at this scale too, but nothing points specifically towards something discoverable at the $\mathrm{LHC}^{1}$. As far as solutions to the hierarchy problem are concerned, Supersymmetry (SUSY) sticks out; the absence of sparticles at the LHC is becoming more and more of a problem, but one should remember that all other theories for new physics have the exact same problem, and hence it is fair to say that SUSY is still our best bet for the future, although it is getting more likely that nothing new will show up and we have misunderstood something fundamental.

One necessary ingredient in the superpotential of SUSY models, is a $\mu$ term, $\mu \widehat{H}_{u} \widehat{H}_{d}$, where $\mu$ is a dimensional parameter that needs to be at the EW scale in order for the EW symmetry breaking to work. Although $\mu$ is far from the only dimensional parameter in SUSY theories, it is special as it respects SUSY; all other mass parameters are soft SUSY breaking terms.

This poses a problem, why should $\mu$, that has nothing to do with SUSY breaking, have the same scale as the SUSY breaking terms?

One solution is offered by the Next to Minimal SUSY SM (NMSSM) [1]; forbid the $\mu \widehat{H}_{u} \widehat{H}_{d}$ term and instead introduce a singlet complex scalar field $S$ and the term, $\lambda \widehat{S} \widehat{H}_{u} \widehat{H}_{d}$. By giving $S$ a VEV - which comes from soft SUSY breaking terms and is hence naturally at the EW scale we then get an effective $\mu$ term, $\lambda\langle S\rangle H_{u} H_{d}$.

The additional singlet superfield introduces two terms into the superpotential,

$$
W_{\mathrm{NMSSM}} \supset \lambda \widehat{S}_{\hat{H}_{u}} \widehat{H}_{d}+\frac{\kappa}{3} \widehat{S}^{3},
$$

where $\lambda$ and $\kappa$ are dimensionless coupling constants.

In addition the soft SUSY breaking potential needs to be supplemented with,

$$
V_{\mathrm{soft}}^{\mathrm{NMSM}} \supset m_{S}^{2}|S|^{2}+\left(\lambda A_{\lambda} H_{u} H_{d} S+\frac{\kappa}{3} A_{\kappa} S^{3}+\text { h.c. }\right),
$$

where $m_{S}, A_{\lambda}$ and $A_{\kappa}$ are dimensionful mass and trilinear parameters. The singlet mass term, $m_{S}^{2}|S|^{2}$ is traded for an effective $\mu$ term and hence the set of parameters relevant for the scalar sector used in the following scans are, $\lambda, \kappa, \mu_{\text {eff }}, \tan \beta, A_{\lambda}$ and $A_{\kappa}$, where $A_{\kappa}$ in some scans have been replaced by the diagonal entry of the pseudoscalar mass matrix, $M_{p}$, more details on the scan procedures can be found in [2].

\section{Light pseudoscalars}

Even though many parameters do affect the pseudoscalar mass, it is mostly driven by $A_{\kappa}$. Since $A_{\kappa}$ is basically unconstrained by other observables, we can put it close to zero in order to

\footnotetext{
${ }^{1}$ But what about the WIMP miracle you may ask, does it not point to EW scale dark matter? Maybe, but there are many other perfectly fine possibilities for dark matter and WIMPs may not necessarily show up at the LHC either. Also the popularity of the WIMP is related to the expectation of new physics at the EW scale due to the hierarchy problem.
} 
get a light pseudoscalar. This means that in most of parameter space, a light pseudoscalar is easily achieved.

Such light pseudoscalars are always very singlet-like and hence hard to produce, which is why they may have escaped detection so far. The only direct production channel of interest is associated production, $b \bar{b} a_{1}$ with $a_{1}$ being the lightest pseudoscalar, but whether this channel is usable is still an open question [3].

The most promising searches look for pseudoscalars in the decay of other particles, especially heavier scalars. Which scalar one should best assume to start such a chain is not clear, but since the only one actually known to exist is the $h_{125}$, that is a good place to start.

In the following we will therefore look at the channel $g g \rightarrow h_{125} \rightarrow a_{1} a_{1}$.

\section{Limits on $\operatorname{Br}\left(h_{125} \rightarrow a_{1} a_{1}\right)$}

The most important value for this channel is $\operatorname{Br}\left(h_{125} \rightarrow a_{1} a_{1}\right)$, the production cross section $\sigma\left(g g \rightarrow h_{125}\right)$ does not change much throughout the parameter space and the branching ratios for the decay channels of the pseudoscalars are pretty much fixed once the mass is known. $\operatorname{Br}\left(h_{125} \rightarrow\right.$ $a_{1} a_{1}$ ) on the other hand, can vary all the way from 0 to 1 .

Since a large $\operatorname{Br}\left(h_{125} \rightarrow a_{1} a_{1}\right)$ will suppress the other branching ratios of $h_{125}$, the most important experimental constraints here are the signal rate constraints for the $125 \mathrm{GeV}$ Higgs. In NMSSMTools $[4,5]$ these are implemented as three separate constraints on the $Z Z, \gamma \gamma$ and $b \bar{b}$ reduce couplings, taken from Lilith [6]. HiggsSignals [7] on the other hand, does an overall fit to all channels simultaneously.

The result is that NMSSSMTools allows $\operatorname{Br}\left(h_{125} \rightarrow a_{1} a_{1}\right)<0.2$ while HiggsSignals allows $\operatorname{Br}\left(h_{125} \rightarrow a_{1} a_{1}\right)<0.5$ !

Which of these values is most trustworthy is hard to say, so we leave that as is and show both options when comparing to data.
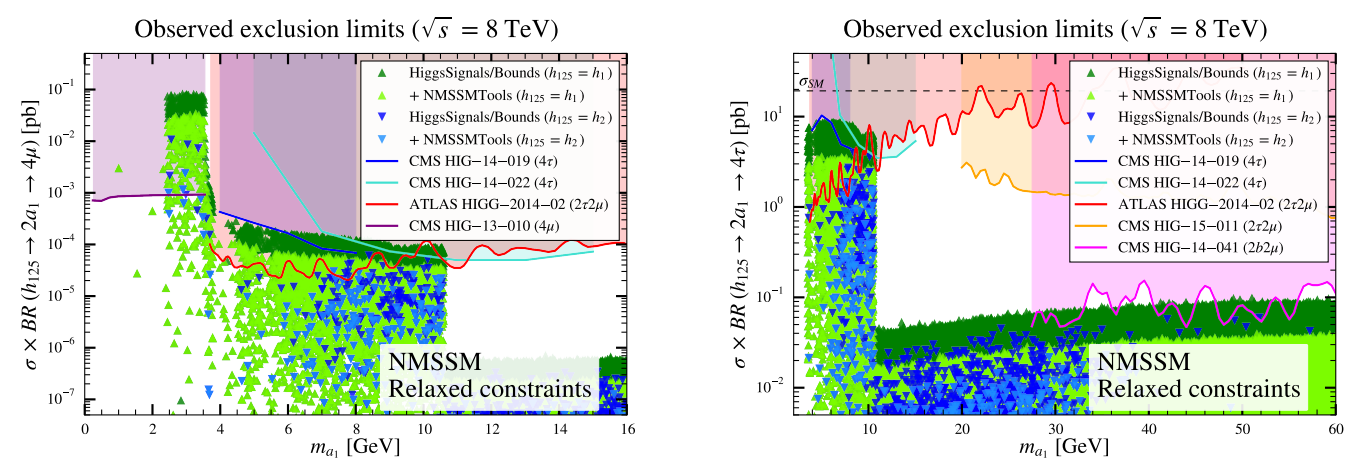

Figure 1: Plots of $\sigma \times B R\left(g g \rightarrow h_{125} \rightarrow 2 a_{1} \rightarrow 4 \mu\right)$ (left) and $\sigma \times B R\left(g g \rightarrow h_{125} \rightarrow 2 a_{1} \rightarrow 4 \tau\right)$ (right) versus $m_{a_{1}}$ for various Higgs assignments in the NMSSM. Dark green/blue points are only required to satisfy Higgs rate constraints from HiggsSignals, whilst lighter green/blue points must also pass NMSSMTools Higgs rate constraints. All points pass a "relaxed" set of constraints, i.e. all other NMSSMTools constraints, but ignoring $(g-2)_{\mu}$ and only an upper limit on relic density. Overlaid are observed exclusion regions from the relevant analyses. 


\section{Experimental limits}

Which final state to look for depends on the pseudoscalar mass. Below $2 m_{\tau}$ looking for muons is rather promising. In the left panel of figure 1, we can see that the $4 \mu$ searches [8] puts serious pressure on the parameter space.

If we move to the range $2 m_{\tau}<m_{a_{1}}<2 m_{b}$, one can still exploit the detectability of muons as well as taus. As can be seen in figure 1, searches for $2 \tau 2 \mu$ [9] are starting to cut into the parameter space, though the impact is not that large yet.

In the higher end, $2 m_{b}<m_{a_{1}}$, the presence of $b$-quarks in the final state makes detection harder, but from the right panel of figure 1 , we can see that $2 b 2 \mu$ searches [10] are starting to have an impact, at least if we accept $\operatorname{Br}\left(h_{125} \rightarrow a_{1} a_{1}\right)$ up to 0.5 as HiggsSignals does.

All in all there is an impressive experimental effort to constrain also these low mass new states.

\section{Conclusions}

Light scalars and pseudoscalars are possible in well motivated theories for new physics, especially in the NMSSM. This presents a different kind of challenge for the LHC experiments as compared to the standard heavy new physics.

Since such light particles would interact very weakly, they can easily have escaped detection and for the same reason will be hard to find at the LHC. One of our best options to find them, is by looking at cascade decays of heavier particles, especially looking for $h_{125} \rightarrow a_{1} a_{1}$.

While these searches are difficult because of the soft final states and the fact that pseudoscalars above $10 \mathrm{GeV}$ mostly decays to $b$-quarks, both ATLAS and CMS are making progress towards constraining this parameter space and some of the low mass searches are already excluding regions of parameter space.

\section{References}

[1] U. Ellwanger, C. Hugonie, and A. M. Teixeira, Phys. Rept. 496 (2010) 1-77, [0 910 . 1785].

[2] R. Aggleton, D. Barducci, N. E. Bomark, S. Moretti and C. Shepherd-Themistocleous, JHEP 02 (2017) 035, [1609.06089].

[3] N.-E. Bomark, S. Moretti, S. Munir, and L. Roszkowski, JHEP 02 (2015) 044, [1 409 . 8393].

[4] U. Ellwanger and C. Hugonie, Comput. Phys. Commun. 175 (2006) 290-303, [hep-ph / 0508022 ].

[5] G. Belanger, F. Boudjema, C. Hugonie, A. Pukhov, and A. Semenov, JCAP 0509 (2005) 001, [hep-ph/0505142].

[6] J. Bernon and B. Dumont, Eur. Phys. J. C75 (2015), no. 9 440, [1502. 04138$].$

[7] P. Bechtle, S. Heinemeyer, O. Stål, T. Stefaniak, and G. Weiglein, Eur. Phys. J. C74 (2014), no. 2 2711, [1305.1933].

[8] CMS Collaboration, V. Khachatryan et. al., Phys. Lett. $B 752$ (2016) 146-168, [1506. 00424 ].

[9] ATLAS Collaboration, G. Aad et. al., Phys. Rev. D92 (2015), no. 5 052002, [1505. 01609].

[10] CMS Collaboration, Tech. Rep. CMS-PAS-HIG-14-041, CERN, Geneva, 2016. 